\title{
Regularisation of 3D Signed Distance Fields
}

\author{
Rasmus R. Paulsen, Jakob Andreas Bærentzen, and Rasmus Larsen \\ Informatics and Mathematical Modelling, Technical University of Denmark \\ Richard Petersens Plads, Building 321, DK-2800 Kgs. Lyngby, Denmark \\ $\{$ rrp $, j a b, r l\} @ i m m . d t u . d k$
}

\begin{abstract}
Signed 3D distance fields are used a in a variety of domains. From shape modelling to surface registration. They are typically computed based on sampled point sets. If the input point set contains holes, the behaviour of the zero-level surface of the distance field is not well defined. In this paper, a novel regularisation approach is described. It is based on an energy formulation, where both local smoothness and data fidelity are included. The minimisation of the global energy is shown to be the solution of a large set of linear equations. The solution to the linear system is found by sparse Cholesky factorisation. It is demonstrated that the zero-level surface will act as a membrane after the proposed regularisation. This effectively closes holes in a predictable way. Finally, the performance of the method is tested with a set of synthetic point clouds of increasing complexity.
\end{abstract}

\section{Introduction}

A signed 3D distance field is a powerful and versatile implicit representation of $2 \mathrm{D}$ surfaces embedded in 3D space. It can be used for a variety of purposes as for example shape analysis [15, shape modelling [2, registration [9], and surface reconstruction [13. A signed distance field consists of distances to a surface that is therefore implicitly defined as the zero-level of the distance field. The distance is defined to be negative inside the surface and positive outside. The inside-outside definition is normally only valid for closed and non-intersecting surfaces. However, as will be shown, the applied regularisation can to a certain degree remove the problems with non-closed surfaces. Commonly, the distance field is computed from a sampled point set with normals using one of several methods [141. However, a distance field computed from a point set is often not well regularised and contains discontinuities. Especially, the behaviour of the distance field can be unpredictable in areas with sparse sampling or no points at all. It is desirable to regularise the distance field so the behaviour of the field is well defined even in areas with no underlying data. In this paper, regularisation is done by applying a constrained smoothing operator to the distance field. In the following, it is described how that can be achieved.

\section{Data}

The data used is a set of synthetic shapes represented as point sets, where each point also has a normal. It is assumed that there are consistent normal directions

A.-B. Salberg, J.Y. Hardeberg, and R. Jenssen (Eds.): SCIA 2009, LNCS 5575, pp. 513 519, 2009.

(C) Springer-Verlag Berlin Heidelberg 2009 
over the point set. There exist several methods for computing consistent normals over unorganised point sets 12 .

\section{Methods}

The signed distance field is represented as a uniform voxel volume. In theory, it is possible to use a multilevel tree-like structure, as for example octrees. However, this complicates matters and is beyond the scope of this work. Initially, the signed distance to the point set is computed using a method similar to the method described in [13]. For each voxel, the five closest (to the voxel centre) input points are found using the standard Euclidean metric. Secondly, the distance to the five points are computed as the projected distance from the voxel centre to the line spanned by the point and its associated normal as seen in Fig. 1] Finally, the distance is chosen as the average of the five distances.

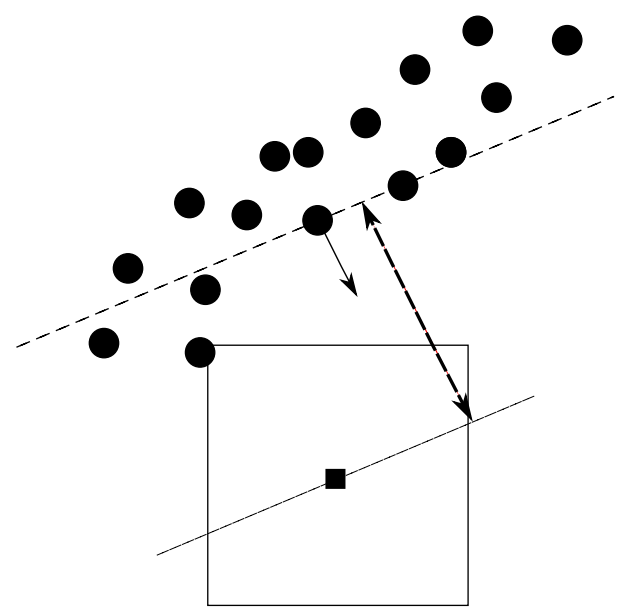

Fig. 1. Projected distance. The distance from the voxel centre (little solid square) to the point with the normal is shown as the dashed double ended arrow.

The zero-level iso-surface can now be extracted using a standard iso-surface extractor as marching cubes [16] or Bloomenthals algorithm [4. However, this surface will neither be smooth nor behave predictable in areas with no input points. This is mostly critical if the input points do not represent shapes that are topologically equivalent to spheres. In the following, marching cubes is used when more than two distinct iso-surfaces exist and the Bloomenthal polygoniser is used if only one surface needs to be extracted.

In order to define the behaviour of the surface, we define an energy function. In this work, we choose a simple energy function based on the difference of 
neighbouring voxels. This classical energy has been widely used in for example Markov Random Fields 3]:

$$
E\left(d_{i}\right)=\frac{1}{n} \sum_{i \sim j}\left(d_{i}-d_{j}\right)^{2}
$$

here $d_{i}$ is the voxel value at position $i$ and $i \sim j$ is the neighbours of the voxel at position $i$. For simplicity a one dimensional indexing system is used instead of the cumbersome $(x, y, z)$ system. In this paper, a 6-neighbourhood system is used, so the number of neighbours are $n=6$, except at the edge of the volume. From statistical physics and using the Gibbs measure it is known that this energy terms induces a Gaussian prior on the voxel values. A global energy for the entire field can now be defined as:

$$
E_{G}=\sum_{i} E\left(d_{i}\right)
$$

Minimising this energy is trivial. It is equal to diffusion and it can therefore be done by convolving the volume using Gaussian kernels. However, the result would be a voxel volume with the same value (the average) in all voxels. This is obviously not very interesting. In order to preserve the information stored in the point set, the energy term in Eq. (11) is changed to:

$$
E_{C}\left(d_{i}\right)=\alpha_{i} \beta\left(d_{i}-d_{i}^{o}\right)^{2}+\left(1-\alpha_{i} \beta\right) \frac{1}{n} \sum_{i \sim j}\left(d_{i}-d_{j}\right)^{2} .
$$

Here $d_{i}^{o}$ is the original distance estimate in voxel $i, \alpha_{i}$ is a local confidence measure, and $\beta$ is a global parameter that controls overall smoothing. Obviously, $\alpha$ should be one where there is complete confidence in the estimated distance and zero where maximum regularisation is needed. In this work, we use a simple distance based estimation of $\alpha$. It is based on the Euclidean distance from the voxel centre to the nearest input point $d_{i}^{E}$. Here $\alpha_{i}=1-\min \left(d_{i}^{E} / d_{\max }^{E}, 1\right)$, where $d_{\max }^{E}$ is a user defined maximum Euclidean distance. A sampling density estimate is computed to estimate $d_{\max }^{E}$. The average $\mu_{l}$ and standard deviation $\sigma_{l}$ of the closest point neighbours distances are estimated from the input point set. The distance is calculated by for each point locating the closest point and computing the Euclidean distance between the two. In this work a value of $d_{\max }^{E}=3 \mu_{l}$ was found to be suitable. A discussion of other confidence measures used for data captured using range scanners can be found in [8]. The global regularisation parameter $\beta$ is set to 0.5 . It is mostly useful in case of Gaussian-like noise in the input data.

A global energy can now be defined using the local energy from Eq. (3):

$$
E_{G, C}=\sum_{i} E_{C}\left(d_{i}\right)
$$


The minimisation of this energy is not as trivial as the minimisation of Eq. (2). Initially, it can be observed that the local energy in Eq. (3) is minimised by:

$$
d_{i}=\alpha_{i} \beta d_{i}^{o}+\left(1-\alpha_{i} \beta\right) \frac{1}{n} \sum_{i \sim j} d_{j}
$$

This can be rearranged into:

$$
\frac{n_{i} d_{i}}{1-\alpha_{i} \beta}-\sum_{i \sim j} d_{j}=\frac{n_{i} \alpha_{i} \beta}{1-\alpha_{i} \beta} d_{i}^{o},
$$

If $N$ is the number of voxel in the volume, we now have $N$ linear equations, each with up to six unknowns (six except for the border voxels). It can therefore be cast into to the linear system $\mathbf{A} x=\mathbf{b}$ :

$$
\left[\begin{array}{cccc}
\frac{n_{1}}{1-\alpha_{1} \beta} & -1 & \ldots & -1 \ldots \\
-1 & \frac{n_{2}}{1-\alpha_{2} \beta} & -1 & \ldots \\
\vdots & & & \\
-1 & & & \\
\vdots & & \ddots
\end{array}\right] x=\left[\begin{array}{c}
\frac{n_{1} \alpha_{1} \beta}{1-\alpha_{1} \beta} d_{1}^{o} \\
\frac{n_{2} \alpha_{2} \beta}{1-\alpha_{2} \beta} d_{2}^{o} \\
\vdots \\
\frac{n_{N} \alpha_{N} \beta}{1-\alpha_{N} \beta} d_{N}^{o}
\end{array}\right]
$$

where $x_{i}=d_{i}$ and $\mathbf{A}$ is a sparse tridiagonal matrix with fringes [17] having dimensions $N x N$. The number of neighbours of a voxel determines the number of -1 in each row in $\mathbf{A}$ (normally six). The column indexes of the -1 depend on the ordering of the voxel volume. In our case the index is computed as $i=x_{t}+y_{t} \cdot N_{x}+z_{t} \cdot N_{x} \cdot N_{y}$, where $\left(x_{t}, y_{t}, z_{t}\right)$ are the voxel displacement compared to the current voxel and $\left(N_{x}, N_{y}, N_{z}\right)$ is the volume dimensions. Some special care is needed for edge and corner voxels that do not have six neighbours. Furthermore, $\mathbf{A}$ is symmetric and positive definite. Several approaches to the solution of these types of problems exist. An option is to use the iterative conjugate gradients [11, but due to its $O\left(n^{2}\right)$ complexity, it is not suitable for large volumes [6]. Multigrid solvers are normally very fast, but require problemdependent design decisions [7. An alternative is to use sparse direct Cholesky solvers [5]. A sparse Cholesky solver initially factors the matrix $\mathbf{A}$, such that the solution is found by back-substitution. This is especially efficient in dynamic system where the right hand side changes and the solution can be found extremely efficient by using the pre-factored matrix to do the back substitution. However, this is not the case in our problem, but the sparse Cholesky approach still proved efficient. A standard sparse Cholesky solver (CHOLMOD) is used to solve the system [10. With this approach, the estimation and regularisation of the distance field is done in less than two minutes for a final voxel volume of approximately $(100,100,100)$ on a standard dual core, $2.4 \mathrm{GHz}, 2 \mathrm{~GB}$ RAM PC.

\section{Results}

The described approach has been applied to different synthetically defined shapes. In Figure 2, a sphere that has been cut by one, two and three planes 

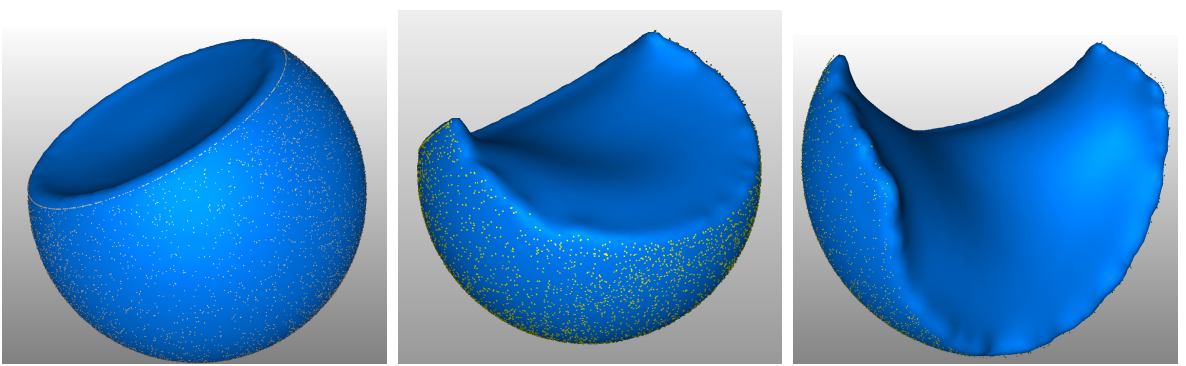

Fig. 2. The zero level iso-surface extracted when the input cloud is a sphere that has one, two, or three cuts
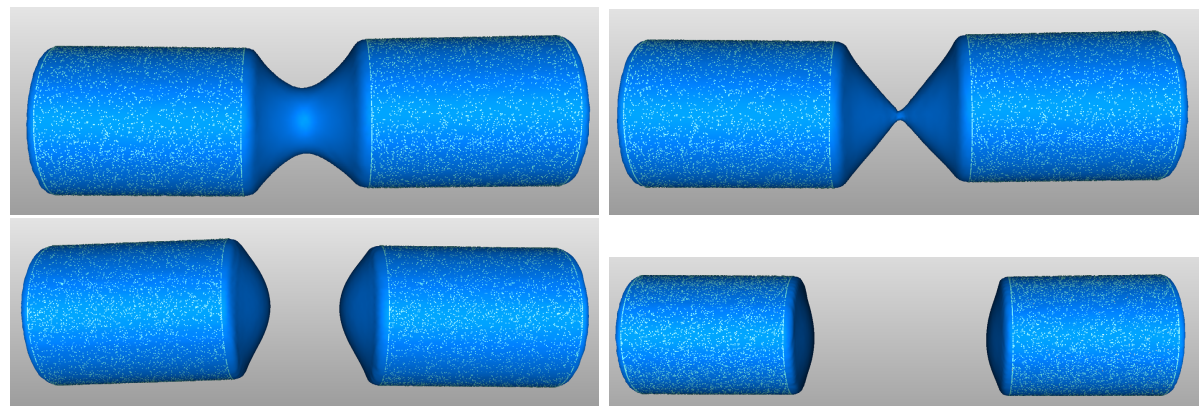

Fig. 3. The zero level iso-surface extracted when the input cloud is two cylinders that are moved away from each other

can be seen. The input points are seen together with the extracted zero-level iso-surface of the regularised distance field.

It can be seen that the zero-level exhibits a membrane-like behaviour. This is not surprising since it can be proved that Eq. (1) is a discretisation of the membrane energy. Furthermore, it can be seen that the zero-level follow the input points. This is due to the local confidence estimates $\alpha$.

In Figure 3, the input consists of the sampled points on two cylinders. It is visualised how the zero-level of the regularised distance field behaves when the two cylinders are moved away from each other. When they are close, the iso-surface connects the two cylinders and when they are far away from each other, the iso-surface encapsulates each cylinder separately. Interestingly, there is a topology change in the iso-surface when comparing the situation with the close cylinders and the far cylinders. This adds an extra flexibility to the method, when seen as a surface fairing approach. Other surface fairing techniques uses an already computed mesh [18] and topology changes are therefore difficult to handle.

Finally, the method has been applied to some more complex shapes as seen in Figure 4 . 

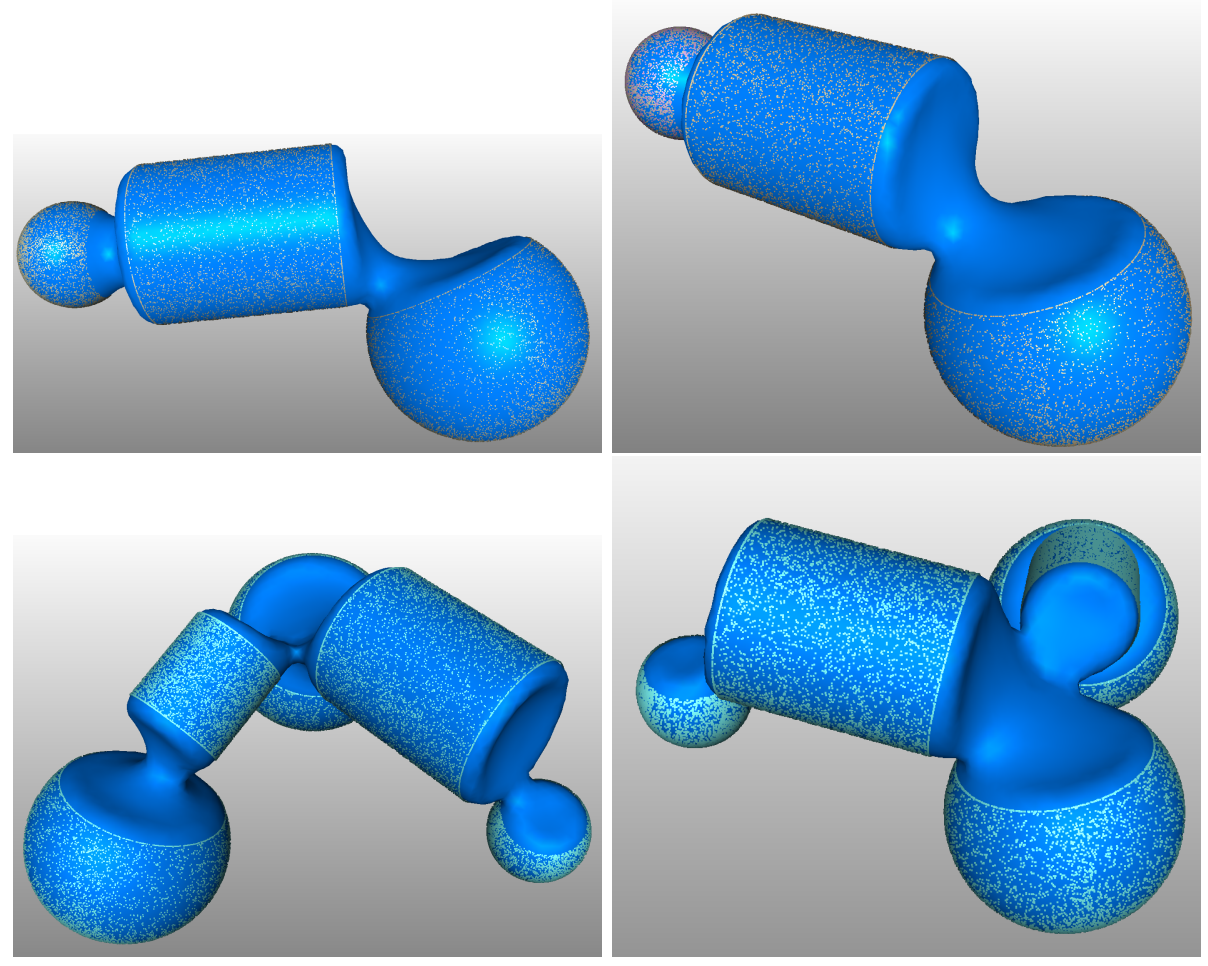

Fig. 4. The zero level iso-surface extracted when the input cloud is complex

\section{Conclusion}

In this paper, a regularisation scheme is presented together with a mathematical framework for fast and efficient estimation of a solution. The approach described can be used for pre-processing distance field before further processing. An obvious use for the approach is surface reconstruction of unorganised point clouds. It should noted, however, that the result of the regularisation in a strict sense is not a distance field, since it will not have global unit gradient length. If a distance field with unit gradient is needed, it can be computed based on the regularised zero-level using one of several update strategies as described in [14].

\section{Acknowledgement}

This work was in part financed by a grant from the Oticon Foundation.

\section{References}

1. Bærentzen, J.A., Aanæs, H.: Computing discrete signed distance fields from triangle meshes. Technical report, Informatics and Mathematical Modelling, Technical University of Denmark, DTU, Richard Petersens Plads, Building 321, DK-2800 Kgs, Lyngby (2002) 
2. Bærentzen, J.A., Christensen, N.J.: Volume sculpting using the level-set method. In: International Conference on Shape Modeling and Applications, pp. 175-182 (2002)

3. Besag, J.: On the statistical analysis of dirty pictures. Journal of the Royal Statistical Society, Series B 48(3), 259-302 (1986)

4. Bloomenthal, J.: An implicit surface polygonizer. In: Graphics Gems IV, pp. 324349 (1994)

5. Botsch, M., Bommes, D., Kobbelt, L.: Efficient Linear System Solvers for Mesh Processing. In: Martin, R., Bez, H.E., Sabin, M.A. (eds.) IMA 2005. LNCS, vol. 3604, pp. 62-83. Springer, Heidelberg (2005)

6. Botsch, M., Sorkine, O.: On Linear Variational Surface Deformation Methods. IEEE Transactions on Visualization and Computer Graphics, 213-230 (2008)

7. Burke, E.K., Cowling, P.I., Keuthen, R.: New models and heuristics for component placement in printed circuit board assembly. In: Proc. Information Intelligence and Systems, pp. 133-140 (1999)

8. Curless, B., Levoy, M.: A volumetric method for building complex models from range images. In: Proceedings of ACM SIGGRAPH, pp. 303-312 (1996)

9. Darkner, S., Vester-Christensen, M., Larsen, R., Nielsen, C., Paulsen, R.R.: Automated 3D Rigid Registration of Open 2D Manifolds. In: MICCAI 2006 Workshop From Statistical Atlases to Personalized Models (2006)

10. Davis, T.A., Hager, W.W.: Row modifications of a sparse cholesky factorization. SIAM Journal on Matrix Analysis and Applications 26(3), 621-639 (2005)

11. Golub, G.H., Van Loan, C.F.: Matrix Computations. Johns Hopkins University Press (1996)

12. Hoppe, H., DeRose, T., Duchamp, T., McDonald, J., Stuetzle, W.: Surface reconstruction from unorganized points. In: ACM SIGGRAPH, pp. 71-78 (1992)

13. Jakobsen, B., Bærentzen, J.A., Christensen, N.J.: Variational volumetric surface reconstruction from unorganized points. In: IEEE/EG International Symposium on Volume Graphics (September 2007)

14. Jones, M.W., Bærentzen, J.A., Sramek, M.: 3D Distance Fields: A Survey of Techniques and Applications. IEEE Transactions On Visualization and Computer Graphics 12(4), 518-599 (2006)

15. Leventon, M.E., Grimson, W.E.L., Faugeras, O.: Statistical shape influence in geodesic active contours. In: IEEE Conference on Computer Vision and Pattern Recognition, 2000, vol. 1 (2000)

16. Lorensen, W.E., Cline, H.E.: Marching cubes: A high resolution 3D surface construction algorithm. Computer Graphics (SIGGRAPH 1987 Proceedings) 21(4), 163-169 (1987)

17. Press, W.H., Teukolsky, S.A., Vetterling, W.T., Flannery, B.P.: Numerical recipes in C: the art of scientific computing. Cambridge University Press, Cambridge (2002)

18. Schneider, R., Kobbelt, L.: Geometric fairing of irregular meshes for free-form surface design. Computer Aided Geometric Design 18(4), 359-379 (2001) 